\title{
Talking About The Smokes: summary and key findings
}

\section{Transforming the evidence to guide Aboriginal and Torres Strait Islander tobacco control}

David P Thomas MBBS, PhD, FAFPHM ${ }^{\prime}$

Maureen E Davey MBBS, FAFPHM ${ }^{2}$

Viki L Briggs BA, GDiplndigHlthProm, MPH ${ }^{3}$

Ron Borland $\mathrm{PhD}^{4}$

1 Menzies School of Health Research, Darwin, NT.

2 Aboriginal Health Service, Tasmanian Aboriginal Centre, Hobart, TAS. 3 University of Melbourne, Melbourne, VIC.

4 Cancer Council Victoria, Melbourne, VIC. david.thomas@ menzies.edu.au

On behalf of the Talking About The Smokes project:

David Thomas (Project Leader)

Pele Bennet

Ron Borland

Viki Briggs

Brad Brown

Sharon Bushby

Sophia Couzos

Maureen Davey

Raylene Foster

Jennifer Hunt

Anna Nicholson

Kathryn Panaretto

Jasmine Sarin

Matthew Stevens

Anke van der Sterren

Sharon Wallace

Chanel Webb

Mary-Anne Williams

doi: 10.5694/mjal5.00464
T he baseline cross-sectional results from the Talking About The Smokes project outlined in this supplement (and summarised in the Box) provide the most detailed national evidence yet to guide practice and policy to reduce the harm caused by tobacco smoking among Aboriginal and Torres Strait Islander peoples. The national prevalence of daily smoking in the Aboriginal and Torres Strait Islander population is falling, but at $42 \%$ is still 2.6 times that of other Australians. ${ }^{1}$ Research evidence to guide Aboriginal and Torres Strait Islander tobacco control has been constrained by the uncertainties of generalising from small local research projects or from the large body of research in other populations. There have been competing hypotheses about whether Aboriginal and Torres Strait Islander smoking and quitting behaviour is similar to or different from other populations. These new results suggest many similarities with other populations.

We found the proportion of Aboriginal and Torres Strait Islander daily smokers who want to quit, have made a quit attempt in the past year, live in smoke-free homes and work in smoke-free workplaces is similar to that of the general population. Similar proportions also demonstrate knowledge of the most harmful health effects of smoking and hold negative personal attitudes towards smoking.

But there are also differences. Fewer Aboriginal and Torres Strait Islander daily smokers than other Australians have ever made a quit attempt or sustained a quit attempt for at least a month, and a lower proportion agree that social norms disapprove of smoking. Even though similar proportions agree that nicotine replacement therapy and stop-smoking medicines help smokers to quit, fewer have used these. In contrast, a higher proportion recalled being advised to quit by a health professional in the past year.

There are also differences in smoking and quitting behaviour and beliefs within the Aboriginal and Torres Strait Islander population, although the socioeconomic gradients were not consistent. For example, more employed people than those who were not employed wanted to quit, had ever tried to quit, had sustained a quit attempt for at least a month, knew about the harms of smoking, had a smoke-free home, had been advised to quit and had used nicotine replacement therapy or stop-smoking medicines. But there were no differences by employment status in quit attempts in the past year, recall of exposure to health information or in many of the attitudes towards smoking.

Using this new information, health staff working directly with Aboriginal and Torres Strait Islander smokers can be encouraged to do more, knowing that most of the smokers they see will want to quit, already know that smoking and passive smoking are harmful, and are likely to live in a smoke-free home and have a history of recent quit attempts. They can be confident that their messages will be understood and welcomed, then focus on the more difficult task of helping people to successfully sustain their quit attempts.

Those working in clinics can build on their existing good work in ensuring that most Aboriginal and Torres Strait Islander smokers regularly receive brief advice about smoking cessation. They can encourage more smokers to use evidence-based measures to prevent relapse during their next quit attempt, such as stop-smoking medicines, the telephone Quitline, and quit-smoking courses, clinics and groups.

Those working in health promotion will need to continue to reinforce and enhance social norms about being smoke-free, to encourage quit attempts and to support smokers trying to sustain quit attempts. There is a need for continued mainstream and national social marketing campaigns, especially those that build on the particular salience of Aboriginal and Torres Strait Islander smokers' concerns about the harmful effects of their smoking on others. Local and Aboriginal and Torres Strait Islander campaigns also appear to be useful.

There are also messages for public health professionals, policymakers, funders and managers. They can justify investing health resources in tobacco control, not only because smoking causes $20 \%$ of Aboriginal and Torres Strait Islander deaths, ${ }^{2}$ but also because improvement is clearly possible. Our findings support maintaining an ongoing commitment to a comprehensive approach to Aboriginal and Torres Strait Islander tobacco control, rather than relying excessively on any single strategy or element. Those working directly with Aboriginal and Torres Strait Islander smokers should be made aware of this new evidence and aided in reorienting their practice to accommodate it. One of the specific challenges will be how to efficiently fund targeted social marketing activity, without wasting social marketing resources through too much fragmentation. ${ }^{3}$

Most recent national policy attention has concentrated on the large increase in dedicated funding initiated by the previous federal government through the Tackling Indigenous Smoking program, followed by the announced cuts to this funding and the review of the program in 2014. The information in this supplement is useful to guide the evolution of the program, but also reminds us that this is only part of the story. Aboriginal and Torres Strait Islander smoking is also being tackled through mainstream tobacco control activities (advertising campaigns, pack warnings and plain packaging, and smoke-free 
Key findings from the baseline survey of the Talking About The Smokes project

We interviewed a nationally representative sample of 2522 Aboriginal and Torres Strait Islander people from 35 locations across Australia, including 1643 smokers ( 1392 daily smokers), 78 ex-smokers who had quit $\leqslant 12$ months before, 233 ex-smokers who had quit > 12 months before, and 568 never-smokers.

\section{Quitting (pages S20, S26 and S39)}

- $70 \%$ of smokers want to quit

- $69 \%$ of daily smokers had ever made a quit attempt

- $48 \%$ of daily smokers had made a quit attempt in the past year

- $47 \%$ of daily smokers who had made a quit attempt in the past 5 years had sustained an attempt for at least 1 month

- $70 \%$ of daily smokers who had made a quit attempt in the past 5 years had strong cravings during their most recent quit attempt, and $72 \%$ found it hard to be around smokers

\section{Second-hand smoke (pages S33 and S63)}

- $53 \%$ of daily smokers reported that smoking was never allowed anywhere inside their home

- $88 \%$ of employed daily smokers reported that smoking was not allowed in any indoor area at their workplace

- $77 \%$ of daily smokers agreed that smoking should be banned everywhere (both indoors and outdoors) at Aboriginal communitycontrolled health services, $93 \%$ agreed it should be banned indoors at other Aboriginal organisations, and 51\% agreed it should be banned at outdoor festivals and sporting events

\section{Knowledge of the health effects of smoking and second-hand smoke} (page S45)

- Most daily smokers reported knowing that smoking causes lung cancer (94\%), heart disease (89\%) and low birthweight (82\%), but fewer were aware that it makes diabetes worse (68\%)

- Most daily smokers reported knowing that second-hand smoke is dangerous to non-smokers (90\%) and children (95\%) and that it causes asthma in children (91\%)

\section{Personal attitudes towards smoking (page S51)}

- $78 \%$ of daily smokers agreed that if they had to do it over again, they would not have started smoking
- $81 \%$ of daily smokers agreed that they spend too much money on cigarettes

- $32 \%$ of daily smokers agreed that smoking is an important part of their life

\section{Social norms about smoking (page S57)}

- $62 \%$ of daily smokers agreed that mainstream society disapproves of smoking, and $40 \%$ agreed that their local community leaders disapprove of smoking

- $70 \%$ of daily smokers agreed that there are fewer and fewer places where they feel comfortable smoking

- $90 \%$ of daily smokers agreed that being a non-smoker sets a good example to children

Anti-tobacco health information (page S67)

- $65 \%$ of smokers recalled often noticing pack warning labels in the past month

- $45 \%$ of smokers recalled often noticing anti-tobacco advertising or information in the past 6 months, most commonly on television

- $48 \%$ of smokers recalled ever noticing any targeted advertising or information featuring Aboriginal and Torres Strait Islander people or artwork in the past 6 months, with 16\% noticing advertising or information featuring local people or artwork

\section{Cessation support (pages S73 and S78)}

- $75 \%$ of daily smokers who had seen a health professional in the past year had been advised to quit

- $37 \%$ of daily smokers had ever used nicotine replacement therapy or stop-smoking medicines, and $23 \%$ had used them in the past year

- Among all smokers and ex-smokers who had quit $\leqslant 12$ months before, nicotine patches were most commonly used (24\%), followed by varenicline (11\%) and nicotine gum (10\%)

We also surveyed 645 staff at 31 Aboriginal community-controlled health services, including 374 Aboriginal and Torres Strait Islander staff who had a lower age- and sex-standardised prevalence of smoking compared with a national sample of the Aboriginal and Torres Strait Islander population (page S85). regulation) and activities already incorporated into routine health care (brief advice and individual cessation support).

This is only the beginning of the evidence that will emerge from the Talking About The Smokes project. It was designed primarily as a cohort study, and analyses of the prospective longitudinal data of the 849 recontacted smokers and ex-smokers will enable more definitive causal interpretations. The involvement of Aboriginal and Torres Strait Islander people and the Aboriginal community-controlled health service sector in all aspects of this project will facilitate the translation of the results into improved practices and policies that will reduce the harm caused by smoking in Aboriginal and Torres Strait Islander communities.

Acknowledgements: We acknowledge and thank the participants, staff and management at the participating Aboriginal community-controlled health services. The project was funded by the Australian Government Department of Health.

Competing interests: No relevant disclosures.

Provenance: Commissioned; not externally peer reviewed.

1 Australian Bureau of Statistics. Australian Aboriginal and Torres Strait Islander Health Survey: updated results, 2012-13. Canberra: ABS, 2014. (ABS Cat. No. 4727.0.55.006.)

2 Vos T, Barker B, Stanley L, Lopez AD. The burden of disease and injury in Aboriginal and Torres Strait Islander peoples 2003. Brisbane: School of Population Health, University of Queensland, 2007.

3 Hornik RC, Ramirez AS. Racial/ethnic disparities and segmentation in communication campaigns. Am Behav Sci 2006; 49: 868-884.

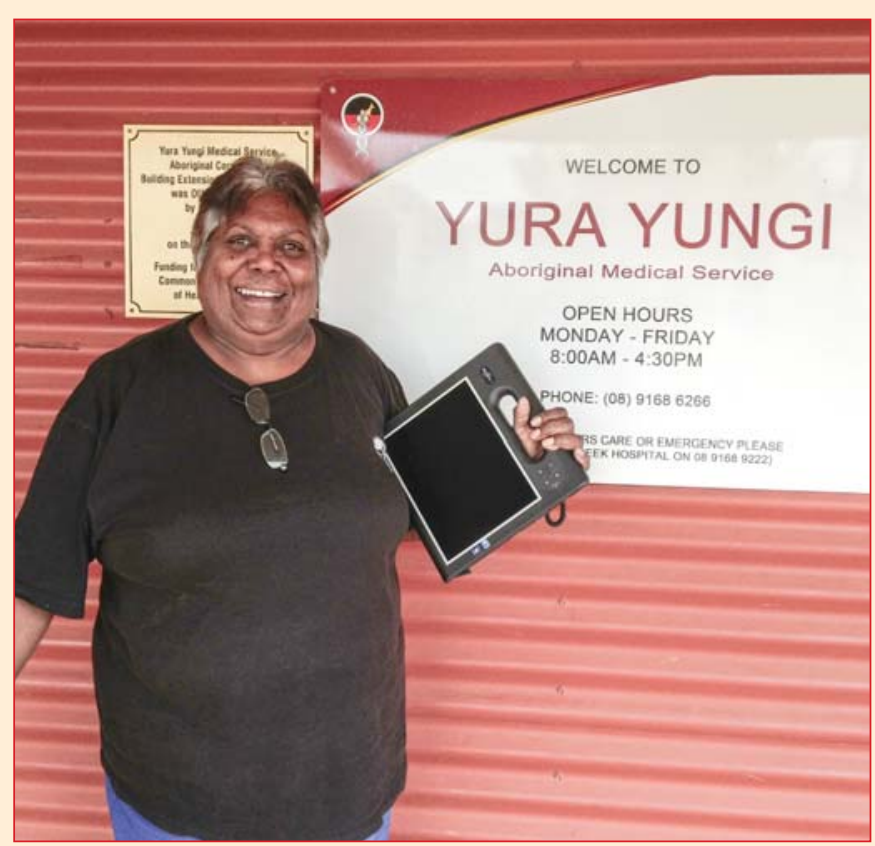

Local research assistant Philomena Lewis at Yura Yungi Medical Service, Halls Creek, WA. 